\title{
The Classification and Diagnostic Algorithm for Primary Lymphatic Dysplasia: an update from 2010 to include molecular findings
}

Fiona Connell1, ${ }^{1}$, Kristiana Gordon ${ }^{2,}$, , Glen Brice ${ }^{3}$, Vaughan Keeley ${ }^{4}$, Steve Jeffery ${ }^{5}$, Peter Mortimer $^{2}$, Sahar Mansour ${ }^{3}$, and Pia Ostergaard ${ }^{5}$

${ }^{1}$ Clinical Genetics, Guy's and St Thomas' NHS Foundation Trust, Guy's Hospital, London SE1 9RT, UK.

2Department of Clinical Science, St George's University of London, London SW17 0RE, UK.

${ }^{3}$ SW Thames Regional Genetics Service, St. George's University of London, London SW17 ORE, UK.

${ }^{4}$ Lymphoedema Clinic, Derby Hospitals NHS Trust, Derby DE22 3NE, UK.

${ }^{5}$ Human Genetics Research Centre, Biomedical Sciences, St George's University of London, London SW17 0RE, UK.

*These two authors contributed equally to this paper.

Correspondence: Dr Sahar Mansour, smansour@sgul.ac.uk

Phone : 02087250957

Fax : 02087253444

\section{Conflict of interest}

All authors declare no conflict of interest.

\section{Acknowledgements}

We would like to acknowledge the members of the Lymphoedema Research Consortium, UK. We extend our thanks to the patients and their families and the British Heart Foundation for funding the work of KG (FS/11/40/28739) and PO (PG/10/58/28477). 


\begin{abstract}
Historically, primary lymphoedema was classified into just three categories depending on the age of onset of swelling; congenital, praecox and tarda. Developments in clinical phenotyping and identification of the genetic cause of some of these conditions have demonstrated that primary lymphoedema is highly heterogenous. In 2010 we introduced a new classification and diagnostic pathway as a clinical and research tool. This algorithm has been used to delineate specific primary lymphoedema phenotypes, facilitating the discovery of new causative genes. This paper reviews the latest molecular findings and provides an updated version of the classification and diagnostic pathway based on this new knowledge.
\end{abstract}

\title{
Keywords
}

Lymphoedema

Generalised lymphatic dysplasia

Phenotyping

FOXC2

GATA2

GJC2

KIF11

VEGFC

FLT4/VEGFR3 


\section{Introduction}

Lymphoedema is chronic swelling due to impaired lymphatic drainage typically affecting the extremities, but may involve any body site. It causes considerable lifelong physical and psychological morbidity; it is disfiguring and results in increased susceptibility to recurrent infection and increased risk of local malignancy $(1,2)$

The lymphatic system is made up of a network of capillaries, collecting vessels and lymphoid organs that serve to maintain fluid homeostasis, immune surveillance and fat absorption from the gut (3). Extravasated interstitial water and proteins are absorbed by lymphatic capillaries and propelled along the lymphatic network by lymph vessel peristalsis or by compression from surrounding skeletal muscles. Lymphoedema is the clinical consequence of swelling within tissue caused by the accumulation of lymphatic fluid due to damage, malfunction, absence or malformation of the lymphatic system.

The lymphatic system may fail for several reasons. The most common scenario is as a result of damage; known as secondary lymphoedema. For example, tumours, cancer treatment (lymphadenectomy, radiotherapy), trauma, venous hypertension and severe infection including filariasis may all damage a previously healthy lymphatic system (47). Alternatively, an individual may develop primary lymphoedema as a result of a genetic predisposition; the lymphatic system fails to develop normally causing impaired drainage of lymph.

Limited data exist on the prevalence of lymphoedema. In 1985 Dale estimated the prevalence of primary lymphoedema as $1 / 6000$ population based upon one UK clinic (8). More recently chronic lymphoedema (of primary and secondary causes) was estimated to affect as many as $1.33 / 1000$ population in the UK (9). A similar survey in Derby estimates 4/1000 (VK, pers. comm.). Further epidemiological studies are needed to establish the true prevalence of primary and secondary lymphoedema.

A review of the literature on primary lymphoedema reveals the term "primary lymphatic dysplasia" used interchangeably with "primary lymphoedema" in several papers (e.g. 2009 Consensus Document of the International Society of Lymphology)(10). We consider lymphatic dysplasia as an inherent developmental abnormality of the lymphatic system resulting in primary lymphoedema. Both terms are used within this review article.

Although the underlying abnormality of the lymphatics is often congenital, with the swelling manifesting as early as the prenatal period, at times it may not develop until puberty or adulthood. The lymphoedema can range from mild swelling to that of grotesque enlargement in chronic cases that have not received adequate treatment. Skin changes may be present, including brawny fibrotic skin and the presence of the KaposiStemmer sign (the failure to pinch/pick up a fold of skin at the base of the second toe as a result of its thickness) that is pathognomonic of lymphoedema (11). Papillomatosis 
(small flesh-coloured papules) occurs as a result of dilatation within the upper dermal lymphatics and subsequent fibrosis of the dermis. Lymphangiectasia appear as small blisters on the skin surface as a result of engorgement of lymphatic vessels. Lymph fluid frequently leaks as a result of minimal trauma and is termed lymphorrhoea.

The mainstay of treatment for lymphoedema is limited to physical therapy in the form of manual lymphatic drainage and compression hosiery/garments in combination with exercise $(12,13)$. Early intervention with skin hygiene advice, appropriate footwear and compression hosiery has beneficial effects on swelling and reducing the risk of complications (e.g. cellulitis) in our paediatric cohort (KG \& PSM, pers. Comm.). There are no effective drug therapies available for the management of primary lymphoedema. Surgical management is largely unsuccessful and is often reserved for use in palliative situations where debulking of tissue is necessary. The use of tumescent liposuction may significantly reduce limb volume in patients who develop increased adipose deposition within their lymphoedematous limb (14). Patient selection prior to liposuction treatment is key to its success; patients must comply with lifelong (all day, every day for life) post-operative compression garments in order to avoid deterioration in lymphoedema.

Mutations in several genes are now recognised to cause lymphatic dysplasia. Some, but not all, of these factors have been demonstrated to play a role in the pathway regulating lymphangiogenesis. Lymphangiogenesis refers to the development of the lymphatic system and begins during embryonic week 6-7 in humans. Recent research has demonstrated that it is regulated by several key factors/proteins (15-17). The first two genes to be identified as causative for human lymphoedema were FLT4 in Milroy disease (MD; MIM 153100) (18-21) and FOXC2 in lymphoedema distichiasis syndrome (LDS; MIM 153400) (22-24). Since then several new genes for human lymphatic disease have been discovered and these will be discussed below.

\section{Classification of primary lymphatic dysplasia}

Allen introduced the first known classification of primary lymphoedemas in 1934 (25). He divided primary lymphoedema into two broad groups: "lymphoedema congenita", i.e. present at birth or shortly thereafter and "lymphoedema praecox" which described cases where swelling occurred after birth. In 1957, Kinmonth et al. added the term "lymphoedema tarda" if the swelling developed after the age of 35 years (26).

It became apparent that this classification system based purely on age-of-onset was over-simplified and redundant in clinical practice as it failed to facilitate categorisation based on more specific phenotypes of primary lymphoedema. In 2010 Connell et al. created a classification pathway founded on clinical phenotype (27). This was based not simply on the age of onset of the lymphoedema but also the sites affected and the presence of associated features e.g. dysmorphology, distichiasis (aberrant eyelashes), varicose veins, prominent large calibre veins, vascular malformations, and limb 
overgrowth. Systemic lymphatic involvement is taken into consideration i.e. a history of hydrops fetalis in utero (the abnormal accumulation of fluid in two or more fetal compartments, including ascites, pleural effusion, pericardial effusion, and subcutaneous oedema (28)), pericardial or pleural effusions, chylous ascites, or gastrointestinal lymphangiectasia. A family history of lymphoedema with determination of the mode of inheritance is an important consideration in this classification.

Specific phenotyping facilitates the identification of subgroups of patients with the same type of primary lymphoedema. This leads to better understanding of the natural history and management of the specific conditions and more accurate recurrence risks for future offspring/generations. These cohorts can then be used for molecular studies to identify causal genes. This has been a fruitful exercise, leading to the identification of several genes, which will be discussed.

\section{Use of the classification pathway}

We have revised and updated the Connell et al. 2010 algorithm (see Fig. 1) and included the newly identified genes. In this review paper, we will focus on the subgroups which now have genes assigned to them, and newly identified conditions based on the clinical presentation. Some of the subgroups in the algorithm will not be covered in this paper as no genes have yet been identified and so the phenotype remains unchanged; the reader can refer to Connell et al. 2010 for descriptions (27). Use of the pathway requires appreciation of certain terminology which is listed in Table 1 and should be read in conjunction with Fig. 1.

The lymphatic dysplasia classification pathway is presented in the form of a colourcoded algorithm to illustrate the five main categories of primary lymphoedema and the individual subtypes within these categories. We appreciate there will be exceptions and outliers to this classification system but for the vast majority of patients it has proved a useful clinical and research tool which is still evolving.

\section{Syndromic (blue section)}

Lymphoedema is a recognised feature of many syndromes. In some of these conditions the lymphoedema is not the primary problem but is an associated feature. The most common conditions in this category include Turner, Noonan (NS; MIM 163950) and Prader Willi (PWS; MIM 176270) syndromes. The genetic causes of many of these syndromes are known and testing is available. A molecular result means that the mode of inheritance can be established and where indicated, prenatal diagnosis can be offered. Table 2 provides a list of syndromes that include lymphoedema as a feature. A new addition to this list is Oculo-dento-digital syndrome (ODD; MIM 164200) which is caused by mutations in the GJA1 gene. Lymphoedema has recently been reported as an associated feature of ODD syndrome in a family, where a novel GJA1 mutation cosegregated with the affected status (33). 
The pathway works in such a way that in conditions in which lymphoedema/lymphatic dysplasia is a main feature, the condition may also be classified elsewhere in the algorithm so as to facilitate the identification of these phenotypes. So although Hennekam (MIM 235510) (34) and Microcephaly-chorioretinopathy-lymphoedemamental retardation(MCLMR; MIM 152950) (35) are syndromes they are discussed in further detail in the 'Generalised lymphatic dysplasia' and 'Congenital lymphoedema' sections respectively.

\section{Systemic/visceral involvement (pink section)}

Individuals with systemic/visceral involvement have a widespread developmental abnormality of the lymphatic system; the swelling is not confined to the peripheries. Onset of lymphatic dysfunction prenatally can present with hydrothoraces or hydrops fetalis. Prenatal hydrops may occur as a result of other conditions (e.g. Parvovirus infection, Rhesus incompatibility, non-immune hydrops due to a number of conditions) and these need to be excluded before considering primary lymphatic dysfunction as the diagnosis. In-utero oedema can lead to dysmorphic facial features in an individual including epicanthic folds, a broad nasal bridge and neck webbing with low set ears (36).

Postnatal signs of systemic lymphatic abnormalities include pericardial and pleural effusions, chylous ascites, and pulmonary and intestinal lymphangiectasia. Patients with intestinal lymphangiectasia often complain of abdominal pain and diarrhoea following the ingestion of fatty foods, however, diagnosis is not always straightforward. Management of these patients is often complex and may include drainage of effusions and medium-chain-triglyceride (MCT) diets to manage intestinal lymphangiectasia and chylous disorders (37). Prompt diagnosis of systemic lymphatic abnormalities aids early intervention that may prove lifesaving in some instances.

Individuals with systemic/visceral involvement can be further divided into two categories: "multisegmental lymphatic dysplasia with systemic involvement" (MLDSI) and "generalised lymphatic dysplasia" (GLD) (27).

\subsection{Multisegmental lymphatic dysplasia with systemic involvement (MLDSI)}

MLDSI is characterised by a segmental pattern of lymphoedema affecting different body parts associated with systemic involvement (Fig. 2a-c). It may be associated with hemifacial swelling and conjunctival oedema on the affected side. More than one limb may be affected, but they may be contralateral. Intelligence is normal and there are no associated structural abnormalities. There is a low sibling and offspring recurrence risk and the underlying mechanism is thought to be explained by somatic mosaicism, but this requires further investigation (FC, pers. comm.). 


\subsection{Generalised lymphatic dysplasia (GLD)/Hennekam syndrome}

Patients with GLD have a more uniform, widespread pattern of lymphoedema affecting all segments of the body (Fig. $2 \mathrm{~d}$-e) and often presenting in utero with hydrops fetalis. A number of affected individuals in this cohort have a positive family history of lymphoedema usually suggestive of autosomal recessive inheritance inferring a higher recurrence risk.

Hennekam syndrome (34) is an example of GLD (Fig. 2d). Other phenotypes exist for this subgroup of patients, but Hennekam syndrome remains the only one with an identified causal gene. Hennekam syndrome is also known as lymphoedemalymphangiectasia-mental retardation syndrome. The phenotype comprises lymphoedema of all four limbs, lymphangiectasia (dysplasia of the lymphatic system of the intestines and/or lungs), variable degrees of learning difficulties and characteristic facies (flat face, flat and broad nasal bridge, hypertelorism) (Fig. 2e). Other associated problems include hypothyroidism, glaucoma, seizures, hearing loss and renal abnormalities. In severe cases it may present in the antenatal period with hydrops fetalis, and/or cardiac abnormalities $(38,39)$. Lymphoscintigraphy has rarely been undertaken in this condition but Bellini demonstrated abnormal drainage in the upper and lower limbs and the thoracic duct in one patient (38).

Hennekam syndrome is inherited in an autosomal recessive manner. Mutations in CCBE1 (collagen and calcium binding EGF-domain 1) on chromosome 18q21 have been identified as causal $(40,41)$. However, $C C B E 1$ mutations were only present in $23 \%$ of cases (40), suggesting genetic heterogeneity.

CCBE1 function in humans has yet to be elucidated, but studies in mice and zebrafish confirm that ccbe1 enhances lymphangiogenesis induced by VEGFC and is also necessary for the budding and sprouting of lymphangioblasts $(42,43)$. Nearly all identified $C C B E 1$ mutations have been missense, but one frameshift has been reported $(40,41,44)$.

\section{Disturbed growth and/or cutaneous/vascular anomalies (yellow section)}

Lymphoedema can be seen in conjunction with other vascular anomalies, disorders of growth and cutaneous abnormalities. Identifying the type of malformation and thereby the vessels involved is important for diagnosis and management. The ISSVA Classification for vascular anomalies has been widely adopted to provide a common nosology (45).

This cohort of patients pose a phenotyping challenge as there is overlap in clinical findings and much confusion in the literature. The recent identification of de novo somatic mutations as the underlying mechanism for some of these conditions may promote further understanding. 


\subsection{AKT/PIK3/mTOR pathway}

AKT acts within a molecular pathway that includes the genes, mTOR and PIK3CA. This pathway is critical as a pro-growth and anti-apoptosis facilitator in cancer but mutations in the genes also have other implications. AKT2 has been shown to have a role in lipomatous overgrowth in association with hypoglycaemia and AKT3 mutations cause hemimegalencephaly (46). PIK3CA, PIK3R2 and mTOR have been implicated in a range of disorders associated with somatic overgrowth, vascular malformations, fibroadipose overgrowth, pigmented cutaneous lesions and megalencephaly (46-49). Distinguishing specific phenotypes from within this cohort of patients is difficult, with much confusion surrounding this spectrum of disorders. CLOVES syndrome (congenital lipomatous overgrowth, vascular malformations, epidermal naevi and skeletal abnormalities) (MIM 612918) has been reported as a specific entity caused by somatic mosaicism of activating mutations in PIK3CA (47). The same gene and same mechanism has also been implicated in a few patients with Klippel-Trenaunay syndrome (MIM $149000)$ and with fibroadipose hyperplasia $(47,50)$. Lymphatic malformations can arise as part of these conditions. Further molecular studies, together with careful phenotyping, will facilitate the understanding of this spectrum of disease. Activated PIK3-AKT signalling underpinning the disease process gives rise to potential for therapeutic targets through inhibition of the PIK3/AKT/mTOR pathway (50). It is likely that combined vascular malformations may also arise as a result of somatic mosaicism.

\subsection{Proteus syndrome}

Proteus syndrome (MIM 176920) is an overgrowth disorder characterised by progressive, segmental overgrowth of skin, connective tissue, skeleton and central nervous system. Lymphatic, along with capillary, malformations are the most common vascular changes seen in Proteus syndrome. Manifestations of Proteus syndrome may not be present at birth but develop and progress in infancy. The diagnosis can be established using diagnostic clinical criteria and/or molecular analysis (51). The majority of individuals with clinically confirmed typical Proteus syndrome were identified to have a common activating mutation in AKT1, c.49G>A (p.Glu17Lys), arising as a result of somatic mosaicism. It is hypothesised that germline $A K T 1$ c.49G $>\mathrm{A}$ mutations would be lethal early in embryonic development (52).

\subsection{WILD syndrome}

A phenotype with the acronym WILD (warts, immunodeficiency, lymphoedema, anogenital dysplasia) has been reported (53) which led us to identify patients in our register with similar clinical features. Therefore, they have been included in the updated pathway. They all have extensive, asymmetrical lymphoedema (multisegmental) (Fig. 3a, d) associated with facial and conjunctival oedema (Fig. 3e), swelling of the genitalia, capillary malformation over the chest (Fig. 3c), and epidermal naevi (Fig. 3b). Persistent wart infections with abnormalities in lymphocyte subset values (particularly CD4) are a 
consistent feature of this condition. These patients are all sporadic, suggesting probable somatic mosaicism. The underlying genetic cause has yet to be identified.

\subsection{Congenital multisegmental lymphoedema}

There is a cohort of patients with congenital multisegmental lymphoedema in whom the pattern of lymphatic failure is asymmetrical with limb sparing (i.e. some limbs are normal) but without overgrowth or cutaneous or vascular anomalies. We hypothesise that somatic mosaicism in gene(s) involved in lymphangiogenesis may explain the aetiology of this subtype of congenital primary lymphoedema, so this condition has been highlighted in yellow as it theoretically shares the same mechanism as the other cohorts in section 3.

\section{Congenital onset primary lymphoedema (green section)}

Historically, all cases of congenital onset primary lymphoedema were classified as Milroy disease (MD) but MD has a distinct clinical phenotype and there are several different types of congenital primary lymphoedema.

\subsection{Milroy disease}

MD was first described in 1892 by William Milroy in a family with congenital lower limb lymphoedema that was painless and non-progressive (54). Brice et al. further refined the phenotype by analysis of 71 individuals with MD (55). They summarised the clinical features of MD and as such the phenotype is easily recognisable. The features include symmetrical congenital lymphoedema of the feet (Fig. 4a). Occasionally, onset of swelling may occur in the first year of life. Lymphoedema is typically confined to the feet and ankles, but may reach the knees. Affected individuals have up-slanting "skijump" toenails due to disturbance of the nail bed by oedema. Prominent large calibre veins may be present on the dorsum of the feet and lower legs. Varicose veins, most commonly the long saphenous vein, have been reported in adults with MD, but do not appear to affect the paediatric population (55). A third of affected males have hydroceles (55). Very rarely, MD may present in the antenatal period with hydrops fetalis (56). The swelling may regress and remain confined to the feet, or the hydrops may progress and result in intrauterine death (57).

Lymphoscintigraphy in Milroy disease confirms the failure of initial lymphatic absorption and has been termed a "functional aplasia" of lymphatic vessels (Fig. 5b). Unlike the mouse model (Chy-mouse) (58), in the human the initial lymphatic vessels are present (confirmed on histological examination) but unable to absorb interstitial fluid (59).

Abnormalities within FLT4; gene that encodes the VEGF receptor type 3 (VEGFR3) on chromosome 5q35 are causal of MD $(18,57)$. Mutations in FLT4 are found in $70 \%$ of patients with congenital onset primary lymphoedema affecting bilateral lower limbs (60). Inheritance is autosomal dominant with $85 \%$ penetrance $(18,19)$. De novo 
mutations may occur so a family history is not mandatory (57). Animal models have confirmed the role of VEGFR3 in lymphangiogenesis and studies have demonstrated that mutant VEGFR3 showed impaired kinase activity $(20,61)$. All reported FLT4 mutations to date have occurred within the tyrosine kinase domain (57). However, the exact mechanism of disease is not yet fully understood.

\subsection{Milroy-like lymphoedema}

Individuals with congenital lower limb lymphoedema who screen negative for FLT4 mutations are classified as having "Milroy-like" lymphoedema. Recently a family with congenital lower limb lymphoedema, prominent large calibre veins and hydroceles inherited in an autosomal dominant pattern over three generations has been described. Affected individuals tested negative for FLT4 mutations but were found to have a frameshift mutation in VEGFC on chromosome 4q34 (62). VEGFC is a ligand for VEGFR3 and controls lymphatic sprouting during embryonic development $(42,63)$. Gordon et al. used in vivo overexpression assays in zebrafish to demonstrate that wildtype human VEGFC, but not mutant VEGFC, caused excessive sprouting of venous-lymphatic vessels, confirming that the $V E G F C$ frameshift mutation was causative for the Milroy-like phenotype seen. It is therefore appropriate to consider VEGFC screening in patients with "Milroy-like" lymphoedema.

\subsection{Microcephaly-chorioretinopathy-lymphoedema-mental retardation syndrome}

A syndrome comprising microcephaly and congenital lymphoedema was reported by Leung in 1985 (35). However, it was only recently that Ostergaard et al. identified that mutations in KIF11 on chromosome 10q24 were causative for autosomal dominant microcephaly variably associated with congenital lymphoedema and/or chorioretinopathy: (microcephaly-lymphoedema-chorioretinal dysplasia (MLCRD), and chorioretinal dysplasia-microcephaly-mental retardation (CDMMR)) (64). Now regrouped under the acronym MCLMR (MIM 152950).

Clinical features include microcephaly ( -2 to -7 standard deviations from the mean) (Fig. 4b), congenital lower limb lymphoedema (mimicking Milroy disease) (Fig. 4c), eye abnormalities (chorioretinopathy and/or hypermetropic astigmatism), and mild to moderate learning difficulties (64). Lymphoscintigraphy shows the same pattern that is seen in Milroy disease with a functional aplasia of lymphatic vessels. MCLMR should be considered in all microcephalic patients with congenital bilateral lower limb swelling.

The KIF11 protein product (EG5) is involved in spindle formation in mitosis but is not known to be directly involved in the lymphangiogenesis pathway (64). The mechanism by which KIF11 exerts its influence on the development of lymphoedema remains a mystery. 


\section{Late onset primary lymphoedema (purple section)}

Late onset lymphoedema refers to the development of lymphoedema occurring after the age of one year. This group contains a number of diverse disorders, some with lifethreatening associated diseases (e.g. Emberger syndrome), but they all share the common finding of non-congenital limb swelling.

\subsection{Lymphoedema distichiasis syndrome}

Lymphoedema in association with distichiasis (aberrant eyelashes) was first recognised in 1899 (65). The lymphoedema of the lower limbs (usually bilateral) (Fig. 5c, upper panel) develops in late childhood (usually during puberty) but onset may be congenital or as late as in the $5^{\text {th }}$ decade. Distichiasis is frequently present at birth but rarely presents until childhood. Distichiasis, the presence of aberrant eyelashes arising from the meibomian glands (Fig. 6a, arrows), is sometimes only detectable on specialist examination and therefore frequently missed. These aberrant lashes can be highly symptomatic and cause ocular irritation, photophobia, recurrent infections (styes) and may require ablative treatment. Conversely, some individuals with distichiasis remain asymptomatic. Distichiasis was reported by Brice et al. to be $95 \%$ penetrant in this syndrome (66).

The lymphoedema distichiasis syndrome (LDS) phenotype also includes ptosis (35\%), cleft palate or Pierre-Robin sequence (3\%) and congenital heart disease (8\%) (66). The vast majority of affected individuals have clinical and/or ultrasonographic evidence of incompetent or varicose veins (66). LDS is inherited in an autosomal dominant manner. It occurs as a result of mutations in the FOXC2 gene on chromosome 16q24 (23). Brice et al. demonstrated that mutations can be identified in more than $95 \%$ of cases with typical LDS (66). Many cases are due to small deletions or insertions (67). FOXC2 encodes a transcription factor necessary for the signal transduction pathway ensuring normal development of the lymphatic collecting vessels and valves (68).

Lymphoscintigraphy of affected individuals demonstrates reflux of lymph within the lower limbs as a result of valve failure within the hyperplastic lymphatics (Fig. 5c, lower panel) (69). Similarly, abnormal venous valves lead to early-onset venous reflux in all patients with FOXC2 mutations (70).

\subsection{Meige disease}

Meige disease (MIM 153200) is probably the most prevalent subtype of primary lymphoedema and was originally described in 1898 by Henry Meige (71). It typically presents with lower limb lymphoedema that rarely extends above the knee (Fig. 5d, upper panel). The lymphoedema does not appear in childhood, but in adolescence or adulthood. There are no other associated features of the condition (72).

Lymphoscintigraphy frequently demonstrates deep re-routing of lymph drainage as evidenced by increased uptake of tracer by popliteal lymph nodes and impaired main superficial lymphatic tract filling (Fig. 5d, lower panel) (PSM, pers. comm.). 
Family history is often consistent with an autosomal dominant pattern of inheritance with variable penetrance. Whilst the causal gene of classical Meige disease has yet to be identified, a number of adolescents and adults with GJC2 gene mutations have presented with lymphoedema localised to the lower limbs (see section on 4-limb lymphoedema below). GJC2 should therefore be considered as a candidate gene for some patients historically labelled as having Meige disease.

\subsection{Emberger syndrome}

The association of primary lymphoedema with myelodysplastic syndrome and/or acute myeloid leukaemia was first described by Emberger in 1979 (73) (MIM 614038). Emberger syndrome is sometimes associated with a high frequency sensorineural deafness that may be congenital. Affected individuals develop bilateral or unilateral lower limb +/- genital lymphoedema in childhood, usually after the age of six years (Fig. 6c) (74). Severe cutaneous warts are a common finding, presumably as a result of their immune dysfunction (Fig. 6b). Myelodysplasia develops at any stage and frequently progresses to acute myeloid leukaemia with a high mortality (74). Lymphoscintigraphy has not been routinely performed in this condition, however, Mansour et al. demonstrated a functional hypoplasia of lymphatics within the affected lower limbs of their patients (74).

Mutations in the GATA2 gene on chromosome 3q21are causal and inherited in an autosomal dominant manner but with a high rate of new mutations (75). GATA2 is expressed in lymphatic, vascular and endocardial endothelial cells (76). Mouse studies suggest the programming of lymphatic valve development may be affected as a result of loss of function of GATA2 (77). GATA2 is also involved in the regulation of haematopoiesis; GATA2 mutations have been reported in heritable forms of myelodysplastic syndrome and acute myeloid leukaemia (78) and two overlapping immunodeficiency syndromes, MonoMAC (Hsu et al. 2011) and dendritic cell, monocyte, B and NK lymphoid deficiency (79).

\subsection{Late onset 4-limb lymphoedema}

In 2010, Ferrell et al. reported that mutations in GJC2 were implicated in the development of lymphoedema (80). Ostergaard et al. 2011 provided further information on the phenotype with five pedigrees demonstrating an autosomal dominant pattern of inherited lymphoedema (MIM 613480) (81). Although there was inter-familial variability of clinical signs the overall phenotype was one of late onset lymphoedema affecting the lower limbs or all four limbs (Fig. 6d, e). A significant number of these individuals also had varicose veins (81). Lymphoscintigraphy demonstrates lymphatic tracts that appear normal but when quantification is done there is significantly reduced absorption from tissues by peripheral lymphatics in all four limbs (81). 
We have seen a number of adolescents and adults with clinical evidence of lymphoedema localised to the lower limbs but with lymphoscintigraphic evidence of impaired lymphatic drainage within all four limbs. It remains to be seen whether these individuals progress to developed clinical signs of upper limb lymphoedema over time.

The functional role of GJC2 within the lymphatic system is unclear. GJC2 encodes the connexin 47 protein located on chromosome 1q42. Homozygous mutations in GJC2 have been reported in patients with Pelizaeus-Merzbacher-like disease (MIM 608804), a rare form of CNS demyelination, but none of these patients or their parents were reported to have lymphoedema (82).

\section{Conclusion}

A number of causal genes have now been identified in the pathogenesis of primary lymphoedema, the majority within the last few years. Gene identification helps delineate the phenotype more specifically. It is hoped that the identification of more pathogenic genes will further our knowledge of the aetiopathogenesis of lymphatic disease. In time this may allow for the development of therapeutic interventions.

The identification of the genetic causes of primary lymphoedema provides a molecular diagnostic test for some of the subtypes. However, there are other primary lymphoedema subtypes for which the underlying genetic cause remains elusive. We predict that rigorous phenotyping in combination with recent advances in genetic analysis, e.g. next generation sequencing, will enhance the identification rate of new genes that cause primary lymphoedema. Accurate phenotyping requires a consistent approach to assessing patients, and the clinical pathway detailed here has proved fit for purpose. As with many genetic conditions, as we learn more about the genotype/phenotype correlations our understanding of these lymphatic disorders will evolve. 


\section{Legends}

Fig. 1. Revised classification pathway for primary lymphoedema. FH: family history; +ve: positive; -ve: negative; unilat: unilateral; bilat: bilateral. Text in red indicates suggested genetic test for the subgroup. Please refer to Table 1 for definition of other terminology used in the figure.

Fig. 2. Generalised lymphatic dysplasia and multisegmental lymphatic dysplasia with systemic involvement (pink section). (a-c) 14 year old girl with MLDSI. (a) Lymphoedema of the left arm associated with intestinal lymphangiectasia. (b) Left hemifacial oedema, (c) close up of left arm. (d-e) Four year old girl with GLD (Hennekam syndrome) due to homozygous mutations in CCBE1 (41) demonstrating (d) widespread lymphoedema, ascites, and (e) facial oedema.

Fig. 3. WILD syndrome (yellow section). (a-e) Adult with WILD syndrome. (a) Lymphoedema and vascular malformation of right leg, (b) epidermal naevus (arrows) of left arm, (c) capillary malformation of chest, (d) lymphoedema of left arm associated with immunodeficiency and persistent warts (arrow) and (e) conjunctival oedema (arrows).

Fig. 4. Congenital primary lymphoedema (green section). (a) Milroy disease: twins with bilateral congenital lymphoedema of the lower limbs, predominantly the dorsum of the feet, due to a heterozygous mutation in VEGFR3. (b-c) Microcephaly-chorioretinopathylymphoedema-mental retardation syndrome (MCLMR) due to heterozygous mutation in KIF11. (b) Typical facial features with microcephaly, large ears, and full cheeks. (c) Congenital lymphoedema of the feet with a similar presentation to Milroy disease.

Fig. 5. Clinical phenotypes and corresponding lymphoscintigraphs. Lymphoscintigraphy showing uptake of Technetium-99 in the inguinal lymph nodes (ILN) at 2 hours after injection in: (a) Healthy control. (b) Milroy disease, demonstrating functional aplasia of the lymphatics (i.e. no uptake into the ILN). (c) Lymphoedema distichiasis syndrome with reasonable uptake to the ILN but evidence of marked reflux due to lymphatic valve incompetence. (d) Meige disease, poor uptake with evidence of rerouting via the deep lymphatic channels resulting in uptake within the popliteal lymph nodes.

Fig. 6. Late onset primary lymphoedema (purple section). (a) Distichiasis; aberrant eyelashes (arrows) arising from the meibomian glands in patient with lymphoedema distichiasis syndrome due to a mutation in FOXC2. (b-c) Emberger syndrome due to mutation in GATA2; (b) persistent warts on fingers (arrows), (c) bilateral, lower limb lymphoedema. (d-e) Late onset 4-limb lymphoedema due to heterozygous mutations in GJC2; (d) upper limb lymphoedema, (e) three family members with 4-limb lymphoedema. Notice the variable degree of swellings between family members. 
Table 1. Definition of terminology used in the classification pathway. This table should be used in conjunction with Fig. 1.

\begin{tabular}{|c|c|}
\hline Congenital onset & Onset of lymphoedema before the age of one year. \\
\hline $\begin{array}{l}\text { Cutaneous } \\
\text { manifestations }\end{array}$ & $\begin{array}{l}\text { Naevi/pigmentation variations (e.g. epidermal naevi)/vascular } \\
\text { malformations. }\end{array}$ \\
\hline Distichiasis & Presence of aberrant eyelashes arising from the meibomian glands. \\
\hline Disturbed growth & $\begin{array}{l}\text { Hypertrophy (overgrowth) and hypotrophy of bone or soft tissue } \\
\text { resulting in altered length of a limb or body part. }\end{array}$ \\
\hline KT/KT-like & Klippel - Trenaunay/ Klippel- Trenaunay-like syndrome. \\
\hline Late onset & Swelling presenting after one year of age. \\
\hline Prenatal onset & $\begin{array}{l}\text { Detection of lymphatic abnormality in the prenatal period. Isolated } \\
\text { pedal oedema is excluded from this definition as this may be a } \\
\text { presentation of Milroy disease. }\end{array}$ \\
\hline Segment & $\begin{array}{l}\text { A region of the body affected by lymphoedema (i.e. face, } \\
\text { conjunctiva, genitalia, upper limbs, lower limbs - each constitute } \\
\text { one body part). Multisegmental refers to more than one segment } \\
\text { affected by lymphoedema. Bilateral lower limb swelling is not } \\
\text { considered to be multisegmental lymphoedema. }\end{array}$ \\
\hline Syndromic & A constellation of abnormalities, one of which is lymphoedema. \\
\hline $\begin{array}{l}\text { Systemic } \\
\text { involvement }\end{array}$ & $\begin{array}{l}\text { Systemic lymphatic problems persisting beyond the newborn } \\
\text { period or manifesting at any age thereafter. This includes hydrops } \\
\text { fetalis, chylous ascites, intestinal lymphangiectasia, pleural and } \\
\text { pericardial effusions, and pulmonary lymphangiectasia. }\end{array}$ \\
\hline $\begin{array}{l}\text { Vascular } \\
\text { anomalies }\end{array}$ & Include congenital vascular abnormalities. \\
\hline
\end{tabular}


Table 2. List of known syndromes associated with lymphoedema and the causative gene (or chromosomal abnormality) if known. Further information for each of these genes can be found on the OMIM website.

\begin{tabular}{|c|c|}
\hline Known syndrome & Chromosome/Gene \\
\hline Aagenaes syndrome & Not known \\
\hline $\begin{array}{l}\text { Carbohydrate deficient glycoprotein types } \\
\text { 1a,1b,1h }\end{array}$ & PMM2, PM1, ALG8 \\
\hline Cardio-facio-cutaneous syndrome & KRAS, BRAF, MAP2K1,MAP2K2 \\
\hline CHARGE syndrome & $\mathrm{CDH7}$ \\
\hline Choanal atresia-lymphoedema & PTPN14 \\
\hline $\begin{array}{l}\text { Ectodermal dysplasia, anhidrotic, } \\
\text { immunodeficiency, osteopetrosis and } \\
\text { lymphoedema (OL-EDA-ID syndrome) }\end{array}$ & $I K B K G(N E M O)$ \\
\hline Fabry disease & $G L A$ \\
\hline Hennekam syndrome & CCBE1 \\
\hline Hypotrichosis-lymphoedema-telangiectasia & SOX18 \\
\hline Irons-Bianchi syndrome & Not known \\
\hline $\begin{array}{l}\text { Lymphoedema-myelodysplasia (Emberger } \\
\text { syndrome) }\end{array}$ & GATA2 \\
\hline Macrocephaly-capillary-malformation (MCM) & PIK3CA \\
\hline $\begin{array}{l}\text { Microcephaly-chorioretinopathy- } \\
\text { lymphoedema-mental retardation (MCLMR) }\end{array}$ & KIF11 \\
\hline Mucke syndrome & Not known \\
\hline Noonan syndrome & PTPN11, KRAS, SOS1, and others \\
\hline Oculo-dento-digital syndrome (ODD) & GJA1 \\
\hline $\begin{array}{l}\text { Progressive encephalopathy, } \\
\text { hypsarrhythmia, optic atrophy (PEHO) }\end{array}$ & Not known \\
\hline Phelan McDermid syndrome & $22 \mathrm{q}$ terminal deletion or ring chromosome 22 \\
\hline Prader Willi & 15q11 microdeletion or maternal UPD 15 \\
\hline Thrombocytopenia with absent radius & 1q21.1 microdeletion and $R B M 8 A$ \\
\hline Turner syndrome & $45 \times 0$ \\
\hline Velocardiofacial syndrome & 22q11 microdeletion \\
\hline Yellow Nail syndrome & Not known \\
\hline
\end{tabular}




\section{References}

1. Cox NH. Oedema as a risk factor for multiple episodes of cellulitis/erysipelas of the lower leg: a series with community follow-up. British Journal of Dermatology 2006: 155: 947-950.

2. Durr HR, Pellengahr C, Nerlich A et al. Stewart-Treves syndrome as a rare complication of a hereditary lymphedema. Vasa-Journal of Vascular Diseases 2004: 33: 42-45.

3. Alitalo K. The lymphatic vasculature in disease. Nature Medicine 2011: 17: 13711380.

4. Paskett ED, Dean JA, Oliveri JM et al. Cancer-Related Lymphedema Risk Factors, Diagnosis, Treatment, and Impact: A Review. Journal of Clinical Oncology 2012: 30: 3726-3733.

5. Bull RH, Gane JN, Evans JEC et al. Abnormal lymph-drainage in patients with chronic venous leg ulcers. Journal of the American Academy of Dermatology 1993: 28: 585-590.

6. Chakraborty S, Gurusamy M, Zawieja DC et al. Lymphatic filariasis: Perspectives on lymphatic remodeling and contractile dysfunction in filarial disease pathogenesis. Microcirculation 2012: [Epub ahead of print]: doi: 10.1111/micc.12031.

7. Stoberl C, Partsch H. Erysipelas and lymphedema - egg or hen. Zeitschrift Fur Hautkrankheiten H\&G 1987: 62: 56-62.

8. Dale RF. The inheritance of primary lymphoedema. Journal of Medical Genetics 1985: 22: 274-278.

9. Moffatt CJ, Franks PJ, Doherty DC et al. Lymphoedema: an underestimated health problem. Qjm-an International Journal of Medicine 2003: 96: 731-738.

10. International Society of L. The diagnosis and treatment of peripheral lymphedema. 2009 Concensus Document of the International Society of Lymphology. Lymphology 2009: 42: 51-60.

11. Stemmer R. A clinical sign for the early and differential diagnosis of lymph edema. Vasa 1976: 5: 261-262.

12. Lee B, Andrade M, Bergan J et al. Diagnosis and treatment of primary lymphedema. Consensus Document of the International Union of Phlebology (IUP)-2009 (vol 29, pg 454, 2010). International Angiology 2010: 29: 570-570.

13. Framework L. Best Practice for the Management of Lymphoedema. International Consensus.: MEP Ltd, 2006.

14. Brorson H. From Lymph to Fat: Liposuction as a Treatment for Complete

Reduction of Lymphedema. International Journal of Lower Extremity Wounds 2012: 11: 10-19.

15. Tammela T, Alitalo K. Lymphangiogenesis: Molecular Mechanisms and Future Promise. Cell 2010: 140: 460-476.

16. Norrmen C, Tammela T, Petrova TV et al. Biological Basis of Therapeutic Lymphangiogenesis. Circulation 2011: 123: 1335-1351.

17. Schulte-Merker S, Sabine A, Petrova TV. Lymphatic vascular morphogenesis in development, physiology, and disease. Journal of Cell Biology 2011: 193: 607-618.

18. Ferrell RE, Levinson KL, Esman JH et al. Hereditary lymphedema: evidence for linkage and genetic heterogeneity. Human Molecular Genetics 1998: 7: 2073-2078.

19. Evans AL, Brice G, Sotirova V et al. Mapping of primary congenital lymphedema to the 5q35.3 region. American Journal of Human Genetics 1999: 64: 547-555. 
20. Karkkainen MJ, Ferrell RE, Lawrence EC et al. Missense mutations interfere with VEGFR-3 signalling in primary lymphoedema. Nature Genetics 2000: 25: 153-159.

21. Irrthum A, Karkkainen MJ, Devriendt K et al. Congenital hereditary lymphedema caused by a mutation that inactivates VEGFR3 tyrosine kinase. American Journal of Human Genetics 2000: 67: 295-301.

22. Mangion J, Rahman N, Mansour S et al. A gene for lymphedema-distichiasis maps to 16q24.3. American Journal of Human Genetics 1999: 65: 427-432.

23. Fang JM, Dagenais SL, Erickson RP et al. Mutations in FOXC2 (MFH-1), a forkhead family transcription factor, are responsible for the hereditary lymphedema-distichiasis syndrome. American Journal of Human Genetics 2000: 67: 1382-1388.

24. Brice G, Mansour S, Bell R et al. Analysis of the phenotypic abnormalities in lymphoedema-distichiasis syndrome in 74 patients with FOXC2 mutations or linkage to 16q24. Journal of Medical Genetics 2002: 39: 478-483.

25. Allen E. Lymphoedema of the extremities. Classification, etiology and differential diagnosis: A study of three hundred cases. Arch Intern Med 1934: 54: 606-624.

26. Kinmonth JB, Taylor GW, Tracy GD et al. Primary lymphoedema; clinical and lymphangiographic studies of a series of 107 patients in which the lower limbs were affected. The British journal of surgery 1957: 45: 1-9.

27. Connell F, Brice G, Jeffery $S$ et al. A new classification system for primary lymphatic dysplasias based on phenotype. Clinical Genetics 2010: 77: 438-452.

28. Warsof SL, Nicolaides KH, Rodeck C. Immune and nonimmune hydrops. Clinical Obstetrics and Gynecology 1986: 29: 533-542.

29. Aagenaes O, Sigstad H, Bjornhan.R. Lymphoedema in hereditary recurrent cholestasis from birth. Archives of Disease in Childhood 1970: 45: 690-695.

30. Glade C, van Steensel MAM, Steijlen PM. Hypotrichosis, lymphedema of the legs and acral telangiectasias - new syndrome? European Journal of Dermatology 2001: 11: 515-517.

31. Irons MB, Bianchi DW, Geggel RL et al. Possible new autosomal recessive syndrome of lymphedema, hydroceles, atrial septal defect, and characteristic facial changes. American Journal of Medical Genetics 1996: 66: 69-71.

32. Mucke J, Hoepffner W, Scheerschmidt G et al. Early onset lymphedema, recessive form - a new form of genetic lymphedema syndrome. European Journal of Pediatrics 1986: 145: 195-198.

33. Brice GW OP, Jeffery S, Gordon KD, Mansour S, Mortimer PS. A novel mutation in GJA1 causing oculodentodigital syndrome and primary lymphoedema in a three generation family. submitted.

34. Hennekam RCM, Geerdink RA, Hamel BCJ et al. Autosomal recessive intestinal lymphangiectasia and lymphedema, with facial anomalies and mental-retardation. American Journal of Medical Genetics 1989: 34: 593-600.

35. Leung AKC. Dominantly inherited syndrome of microcephaly and congenital lymphedema. Clinical Genetics 1985: 27: 611-612.

36. Opitz JM. On congenital lymphedema. Am J Med Genet 1986: 24: 127-129.

37. Jeffries GH, Chapman A, Sleisenger MH. Low-fat diet in intestinal lymphangiectasia. Its effect on albumin metabolism. The New England journal of medicine 1964: 270: 761-766.

38. Bellini C, Mazzella M, Arioni C et al. Hennekam syndrome presenting as nonimmune hydrops fetalis, congenital chylothorax, and congenital pulmonary lymphangiectasia. American Journal of Medical Genetics Part A 2003: 120A: 92-96. 
39. Van Balkom IDC, Alders M, Allanson J et al. Lymphedema-lymphangiectasiamental retardation (Hennekam) syndrome: A review. American Journal of Medical Genetics 2002: 112: 412-421.

40. Alders M, Hogan BM, Gjini E et al. Mutations in CCBE1 cause generalized lymph vessel dysplasia in humans. Nature Genetics 2009: 41: 1272-1274.

41. Connell F, Kalidas K, Ostergaard P et al. Linkage and sequence analysis indicate that CCBE1 is mutated in recessively inherited generalised lymphatic dysplasia. Human Genetics 2010: 127: 231-241.

42. Hogan BM, Bos FL, Bussmann J et al. ccbe1 is required for embryonic lymphangiogenesis and venous sprouting. Nature Genetics 2009: 41: 396-398. 43. Bos FL, Caunt M, Peterson-Maduro J et al. CCBE1 Is Essential for Mammalian Lymphatic Vascular Development and Enhances the Lymphangiogenic Effect of Vascular Endothelial Growth Factor-C In Vivo. Circulation Research 2011: 109: 486-491.

44. Connell FC, Kalidas K, Ostergaard P et al. CCBE1 mutations can cause a mild, atypical form of generalized lymphatic dysplasia but are not a common cause of nonimmune hydrops fetalis. Clinical Genetics 2012: 81: 191-197.

45. Enjolras 0, Mulliken JB. Vascular tumors and vascular malformations (new issues). Advances in dermatology 1997: 13: 375-423.

46. Lee JH, My H, Silhavy JL et al. De novo somatic mutations in components of the PI3K-AKT3-mTOR pathway cause hemimegalencephaly. Nature Genetics 2012: 44: 941945.

47. Kurek KC, Luks VL, Ayturk UM et al. Somatic Mosaic Activating Mutations in PIK3CA Cause CLOVES Syndrome. American Journal of Human Genetics 2012: 90: 11081115.

48. Lindhurst MJ, Parker VER, Payne F et al. Mosaic overgrowth with fibroadipose hyperplasia is caused by somatic activating mutations in PIK3CA. Nature Genetics 2012: 44: 928-933.

49. Riviere J-B, Mirzaa GM, O'Roak BJ et al. De novo germline and postzygotic mutations in AKT3, PIK3R2 and PIK3CA cause a spectrum of related megalencephaly syndromes. Nature Genetics 2012: 44: 934-940.

50. Lindhurst MJ, Parker VER, Payne F et al. Mosaic overgrowth with fibroadipose hyperplasia is caused by somatic activating mutations in PIK3CA. Nature Genetics 2012: 44.

51. Biesecker L. The challenges of Proteus syndrome: diagnosis and management. European Journal of Human Genetics 2006: 14: 1151-1157.

52. Lindhurst MJ, Sapp JC, Teer JK et al. A Mosaic Activating Mutation in AKT1 Associated with the Proteus Syndrome. New England Journal of Medicine 2011: 365: 611-619.

53. Kreuter A, Hochdorfer B, Brockmeyer NH et al. A human papillomavirusassociated disease with disseminated warts, depressed cell-mediated immunity, primary lymphedema, and anogenital dysplasia - WILD syndrome. Archives of Dermatology 2008: 144: 366-372.

54. Milroy W. An undescribed variety of hereditary edema. New York Medical Journal 1892: 56: 505-508.

55. Brice G, Child AH, Evans A et al. Milroy disease and the VEGFR-3 mutation phenotype. Journal of Medical Genetics 2005: 42: 98-102. 
56. Ghalamkarpour A, Morlot S, Raas-Rothschild A et al. Hereditary lymphedema type I associated with VEGFR3 mutation: the first de novo case and atypical presentations. Clinical Genetics 2006: 70: 330-335.

57. Gordon K, Spiden SL, Connell FC et al. FLT4/VEGFR3 and Milroy Disease: Novel Mutations, a Review of Published Variants and Database Update. Human mutation 2013: 34: 23-31.

58. Karkkainen MJ, Saaristo A, Jussila L et al. A model for gene therapy of human hereditary lymphedema. Proceedings of the National Academy of Sciences of the United States of America 2001: 98: 12677-12682.

59. Mellor RH, Hubert CE, Stanton AWB et al. Lymphatic Dysfunction, Not Aplasia, Underlies Milroy Disease. Microcirculation 2010: 17: 281-296.

60. Connell FC, Ostergaard $\mathrm{P}$, Carver $\mathrm{C}$ et al. Analysis of the coding regions of VEGFR3 and VEGFC in Milroy disease and other primary lymphoedemas. Human Genetics 2009: 124: 625-631.

61. Kaipainen A, Korhonen J, Mustonen T et al. Expression of the fms-like tyrosine kinase-4 gene becomes restricted to lymphatic endothelium during development. Proceedings of the National Academy of Sciences of the United States of America 1995: 92: 3566-3570.

62. Gordon $\mathrm{K}$, Schulte D, Brice G et al. Mutation in vascular endothelial growth factor-C, a ligand for vascular endothelial growth factor receptor-3, is associated with autosomal dominant milroy-like primary lymphedema. Circulation research 2013: 112: 956-960.

63. Kuchler AM, Gjini E, Peterson-Maduro J et al. Development of the zebrafish lymphatic system requires VEGFC signaling. Current Biology 2006: 16: 1244-1248. 64. Ostergaard P, Simpson MA, Mendola A et al. Mutations in KIF11 Cause Autosomal-Dominant Microcephaly Variably Associated with Congenital Lymphedema and Chorioretinopathy. American Journal of Human Genetics 2012: 90: 356-362.

65. Kuhnt H. IV. Ueber Distichiasis (congenita) vera. Ophthalmologica 1899: 2: 4657.

66. Brice G, Mansour S, Bell R et al. Analysis of the phenotypic abnormalities in lymphoedema-distichiasis syndrome in 74 patients with FOXC2 mutations or linkage to 16q24. Journal of Medical Genetics 2002: 39.

67. Bell R, Brice G, Child AH et al. Analysis of lymphoedema-distichiasis families for FOXC2 mutations reveals small insertions and deletions throughout the gene. Human Genetics 2001: 108: 546-551.

68. Norrmen C, Ivanov KI, Cheng J et al. FOXC2 controls formation and maturation of lymphatic collecting vessels through cooperation with NFATc1. Journal of Cell Biology 2009: 185: 439-457.

69. Petrova TV, Karpanen T, Norrmen C et al. Defective valves and abnormal mural cell recruitment underlie lymphatic vascular failure in lymphedema distichiasis. Nature Medicine 2004: 10: 974-981.

70. Mellor RH, Brice G, Stanton AWB et al. Mutations in FOXC2 are strongly associated with primary valve failure in veins of the lower limb. Circulation 2007: 115: 1912-1920.

71. Meige H. Dystrophie oedemateuse hereditaire. La Presse Medicale 1898: 6: 341343. 
72. Rezaie $\mathrm{T}$, Ghoroghchian R, Bell R et al. Primary non-syndromic lymphoedema (Meige disease) is not caused by mutations in FOXC2. European Journal of Human Genetics 2008: 16: 300-304.

73. Emberger JM, Navarro M, Dejean $M$ et al. Deaf mutism, lymphedema of the lawer limbs and hematological anomalies (acute-leukemia, cytopenia) with autosomal dominant transmission. Journal De Genetique Humaine 1979: 27: 237-245. 74. Mansour S, Connell F, Steward C et al. Emberger Syndrome-Primary Lymphedema With Myelodysplasia: Report of Seven New Cases. American Journal of Medical Genetics Part A 2010: 152A: 2287-2296.

75. Ostergaard P, Simpson MA, Connell FC et al. Mutations in GATA2 cause primary lymphedema associated with a predisposition to acute myeloid leukemia (Emberger syndrome). Nature Genetics 2011: 43: 929-931.

76. Khandekar M, Brandt W, Zhou Y et al. A Gata2 intronic enhancer confers its panendothelia-specific regulation. Development 2007: 134: 1703-1712.

77. Kazenwadel J, Secker GA, Liu YJ et al. Loss-of-function germline GATA2 mutations in patients with MDS/AML or MonoMAC syndrome and primary lymphedema reveal a key role for GATA2 in the lymphatic vasculature. Blood 2012: 119: 1283-1291. 78. Hahn CN, Chong C-E, Carmichael CL et al. Heritable GATA2 mutations associated with familial myelodysplastic syndrome and acute myeloid leukemia. Nature Genetics 2011: 43: 1012-U1130.

79. Dickinson RE, Griffin H, Bigley V et al. Exome sequencing identifies GATA-2 mutation as the cause of dendritic cell, monocyte, B and NK lymphoid deficiency. Blood 2011: 118: 2656-2658.

80. Ferrell RE, Baty CJ, Kimak MA et al. GJC2 Missense Mutations Cause Human Lymphedema. American Journal of Human Genetics 2010: 86: 943-948.

81. Ostergaard P, Simpson MA, Brice G et al. Rapid identification of mutations in GJC2 in primary lymphoedema using whole exome sequencing combined with linkage analysis with delineation of the phenotype. Journal of Medical Genetics 2011: 48: 251255.

82. Orthmann-Murphy JL, Salsano E, Abrams CK et al. Hereditary spastic paraplegia is a novel phenotype for GJA12/GJC2 mutations. Brain, Vol. 132. England, 2009: 426438. 


\section{Figure 1:}

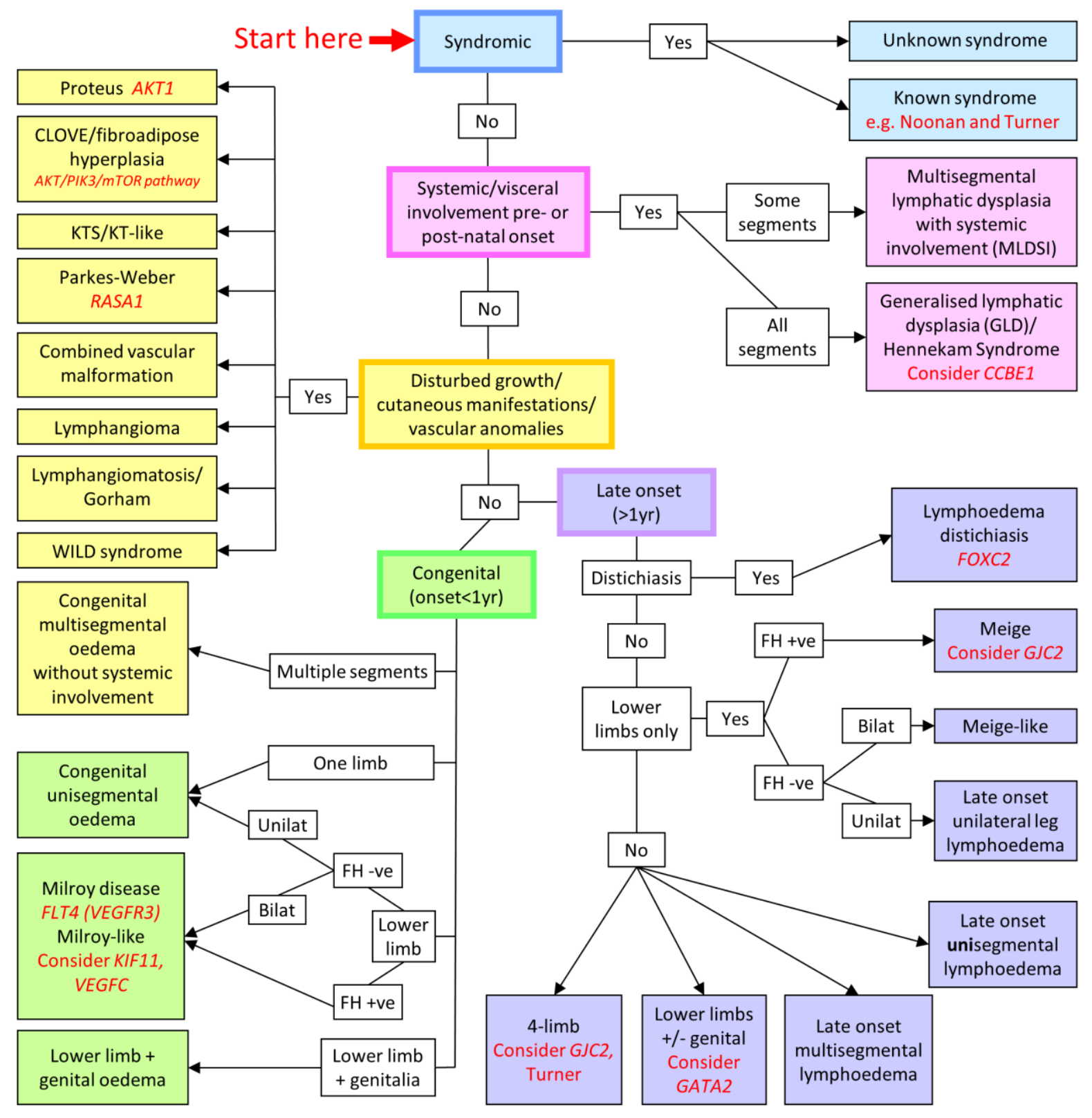


Figure 2:

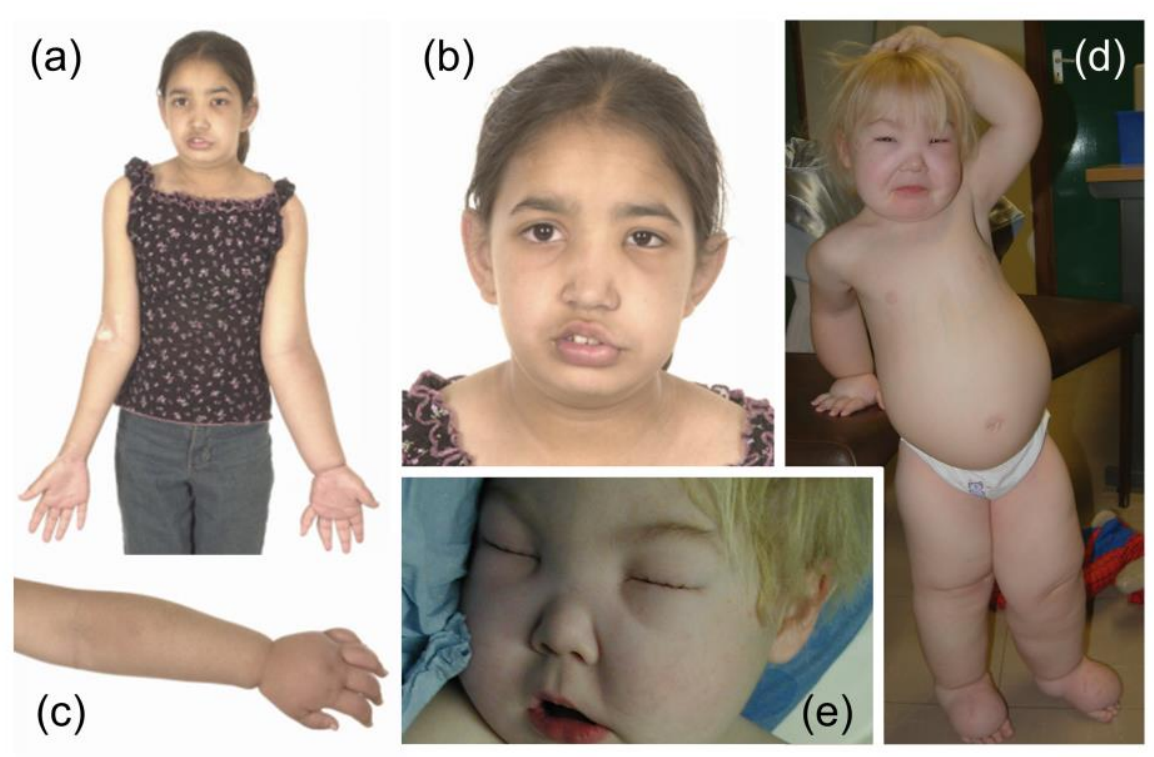


Figure 3:

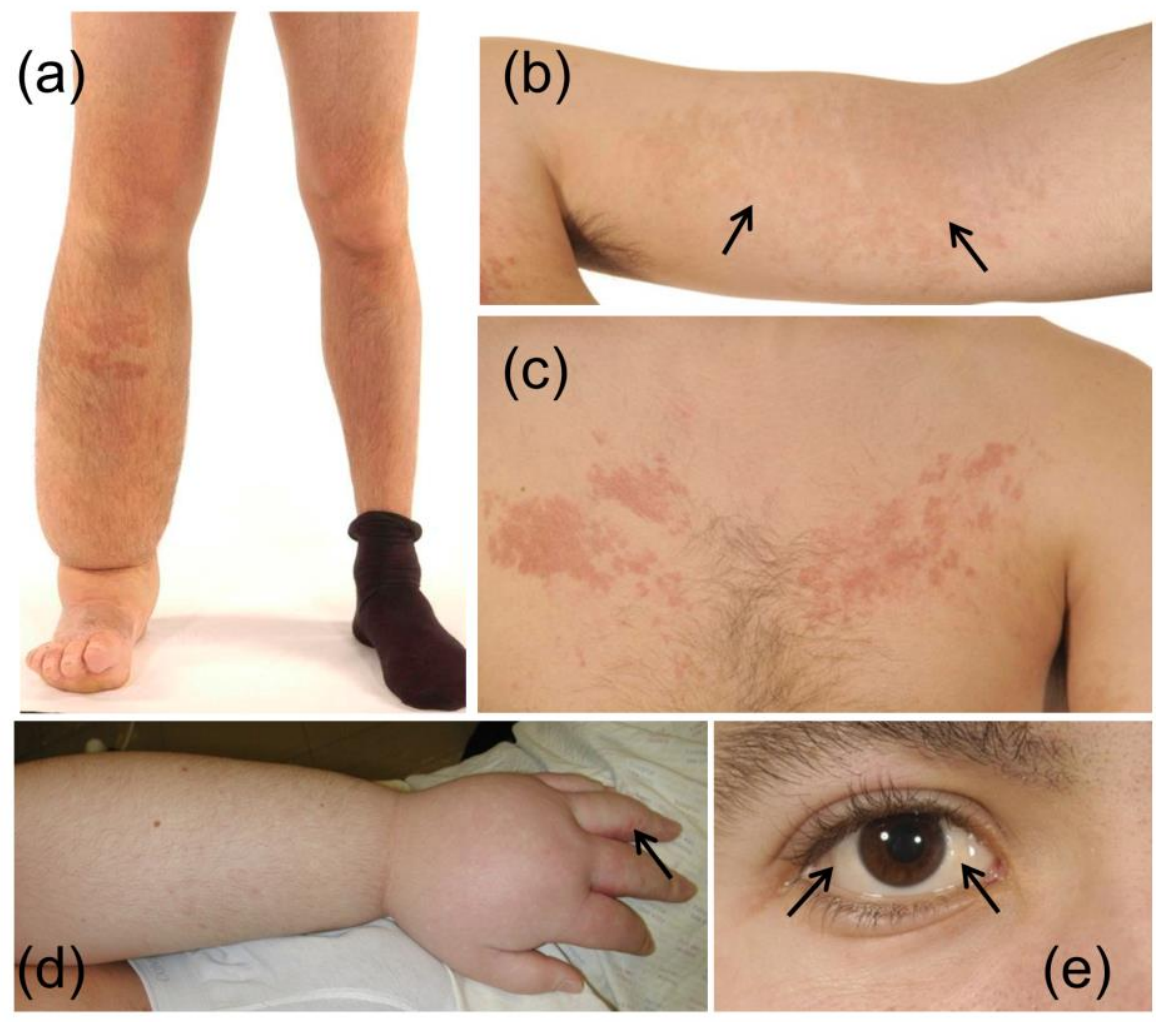


Figure 4:

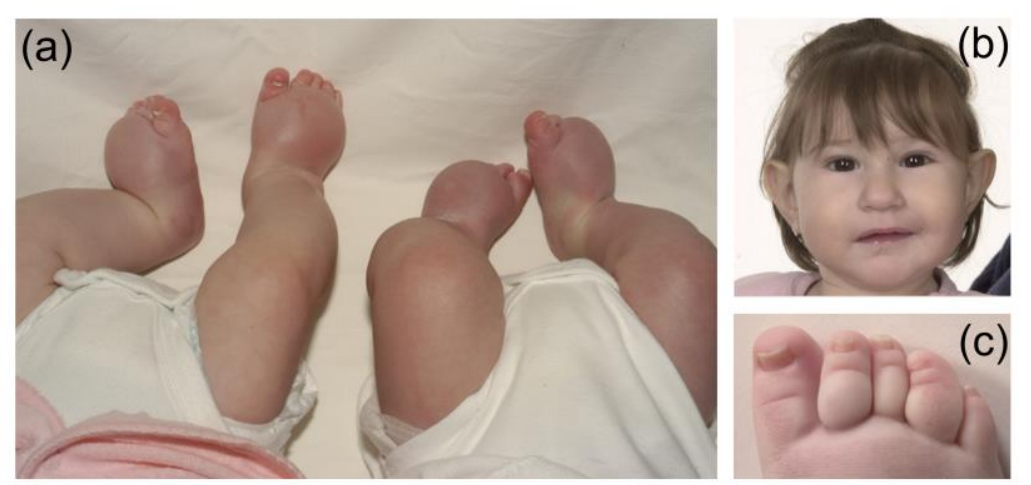


Figure 5:
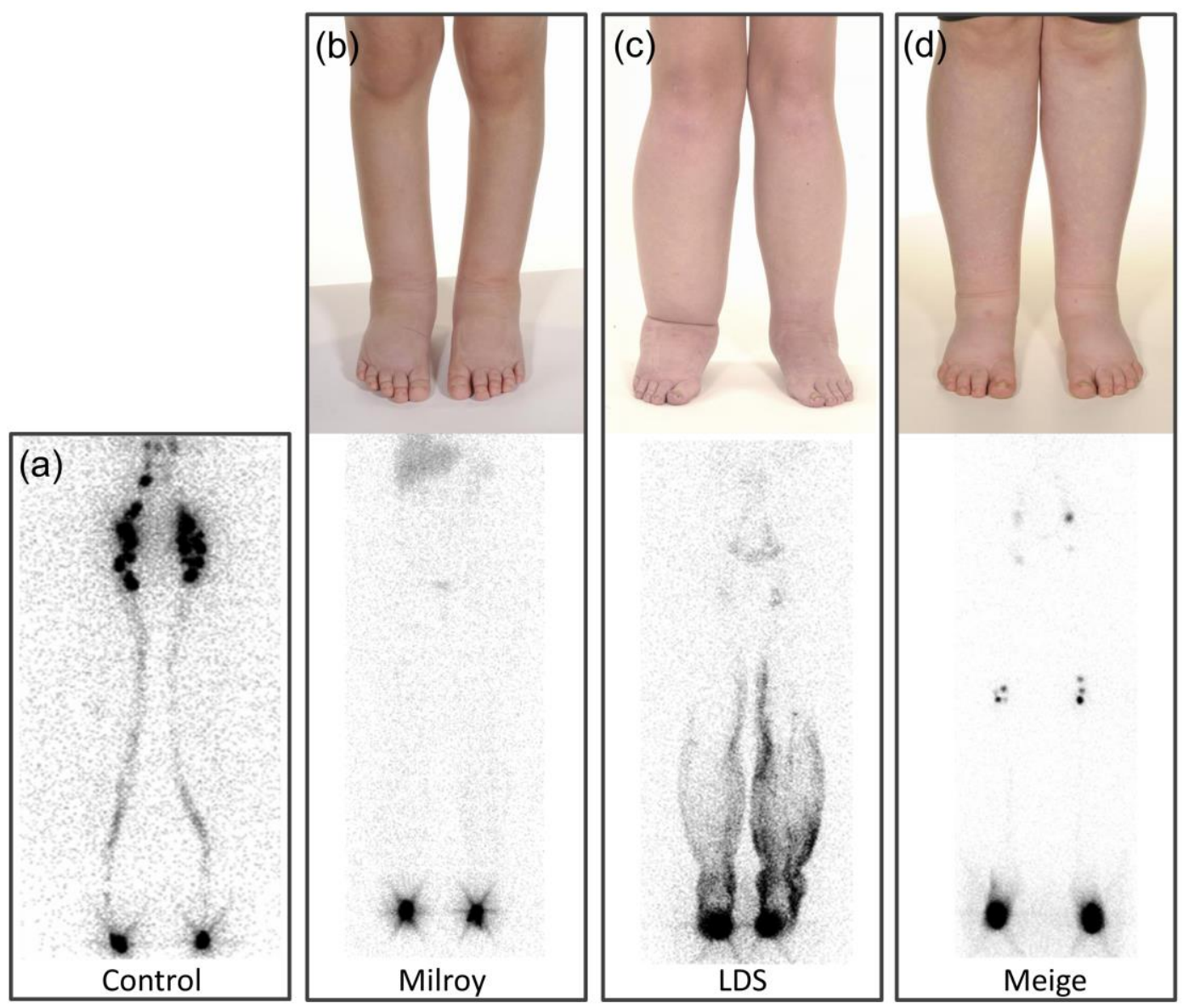
Figure 6:

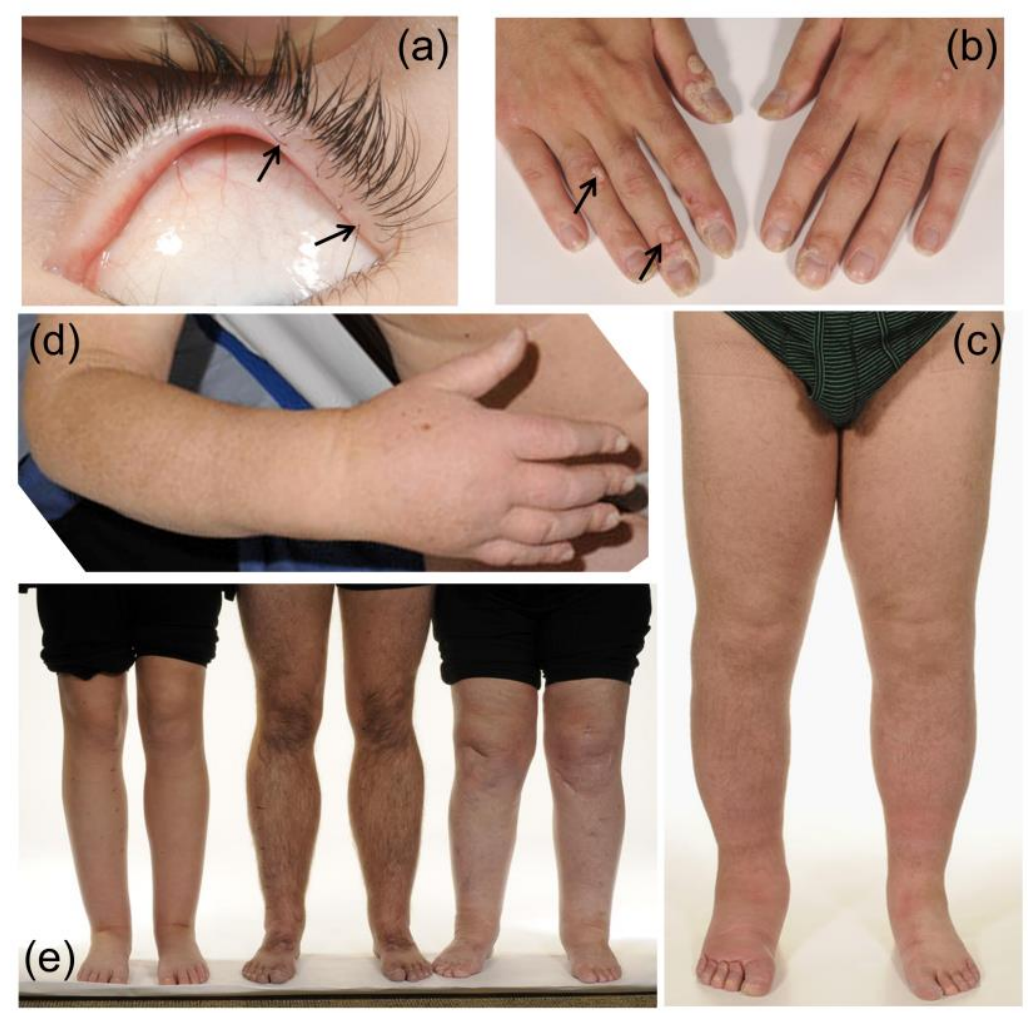

